\title{
Cutting balloon use may ease the optimal apposition of bioresorbable vascular scaffold in in-stent stenosis
}

\author{
Ahmet Karabulut, Yasemin Demirci \\ Department of Cardiology, Faculty of Medicine, Acıbadem University, Acıbadem Atakent Hospital, Istanbul, Turkey
}

Postep Kardiol Inter 2015; 11, 1 (39): 64-66

DOI: $10.5114 /$ pwki.2015.49190

\begin{abstract}
A bstract
Bioresorbable vascular scaffolds (BVS) have different mechanical properties as compared to metallic stents. Therefore, the standard procedural technique to achieve appropriate deployment may differ. Utilisation of debulking techniques, including cutting balloon and directional atherectomy prior to BVS deployment, is still questionable. Herein, we discuss a case of coronary in-stent restenosis and reveal the advantage of predilatation of the lesion with cutting balloon prior to BVS deployment.
\end{abstract}

Key words: cutting balloon, bioresorbable vascular scaffolds, optimisation.

\section{Introduction}

The introduction of bioresorbable vascular scaffold (BVS) started a new era in interventional cardiology [1]. Although the basic deployment techniques of such scaffolds are the same as with metallic stents, because of

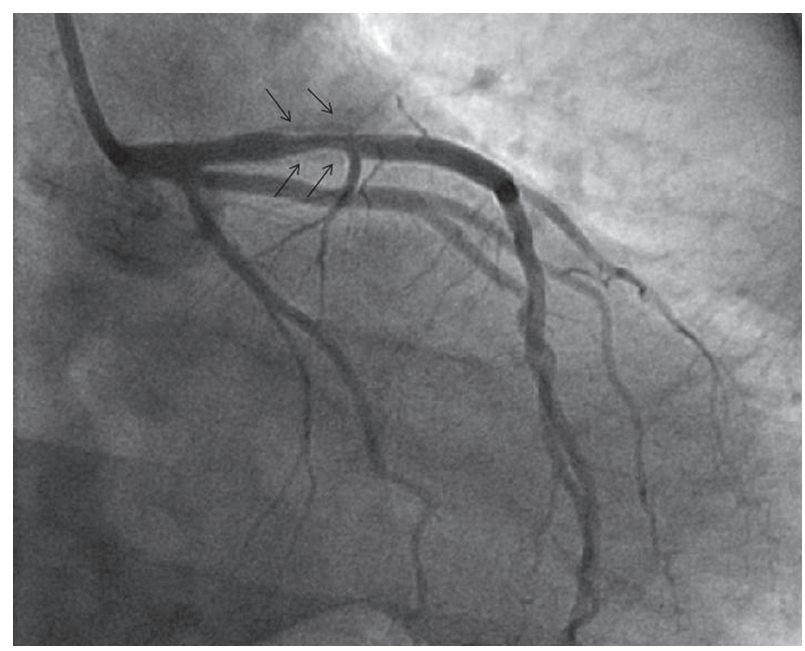

Figure 1. Right anterior oblique caudal view of left coronary arteries. This image shows proximal in-stent stenosis of the left anterior descending artery. Arrows indicate the edge of previous stents the relative restriction in post dilatation, preparation of the lesion before the deployment of the BVS is a critical issue.

Herein, we discus a case of coronary in-stent restenosis and reveal the advantage of predilatation of the lesion with a cutting balloon prior to BVS deployment.

\section{Case report}

A 29-year-old male patient presented with typical angina on exertion. In his medical background, coronary stenting $(4.0 \times 12 \mathrm{~mm}$ bare metal stent (BMS)) had been performed to the proximal left anterior descending artery (LAD) 2 years previously. Due to stent restenosis, another BMS $(4.0 \times 12 \mathrm{~mm})$ was deployed to an in-stent lesion a year later. The patient was followed with aspirin, clopidogrel, statin, and angiotensin-converting enzyme (ACE) inhibitor. In the final presentation, left ventricular ejection fraction was normal and exercise stress test suggested coronary ischaemia. Coronary angiography showed diffuse in-stent $70-75 \%$ stenosis in the LAD (Figure 1). Subsequently, coronary intervention with BVS was scheduled. Cutting balloon was preferred for predilation of the lesion due to extensive diffuse disease and the presence of two overlapping BMSs that could resist the expansion of the new stent/scaffold. After crossing of the lesion, predilatation was performed with $3.5 \times 15$ cutting

\section{Corresponding author:}

Ahmet Karabulut MD, Assoc. Prof., Department of Cardiology, Acıbadem Atakent Hospital, Halkalı Merkez Mah. Turgut Özal Bulvarı, No: 16, 34303, Kucukcekmece, Istanbul, Turkey, phone: +90 5053577477, fax: +90 2124044445, e-mail: drkarabulut@yahoo.com Received: 15.05.2014, accepted: 17.12.2014. 

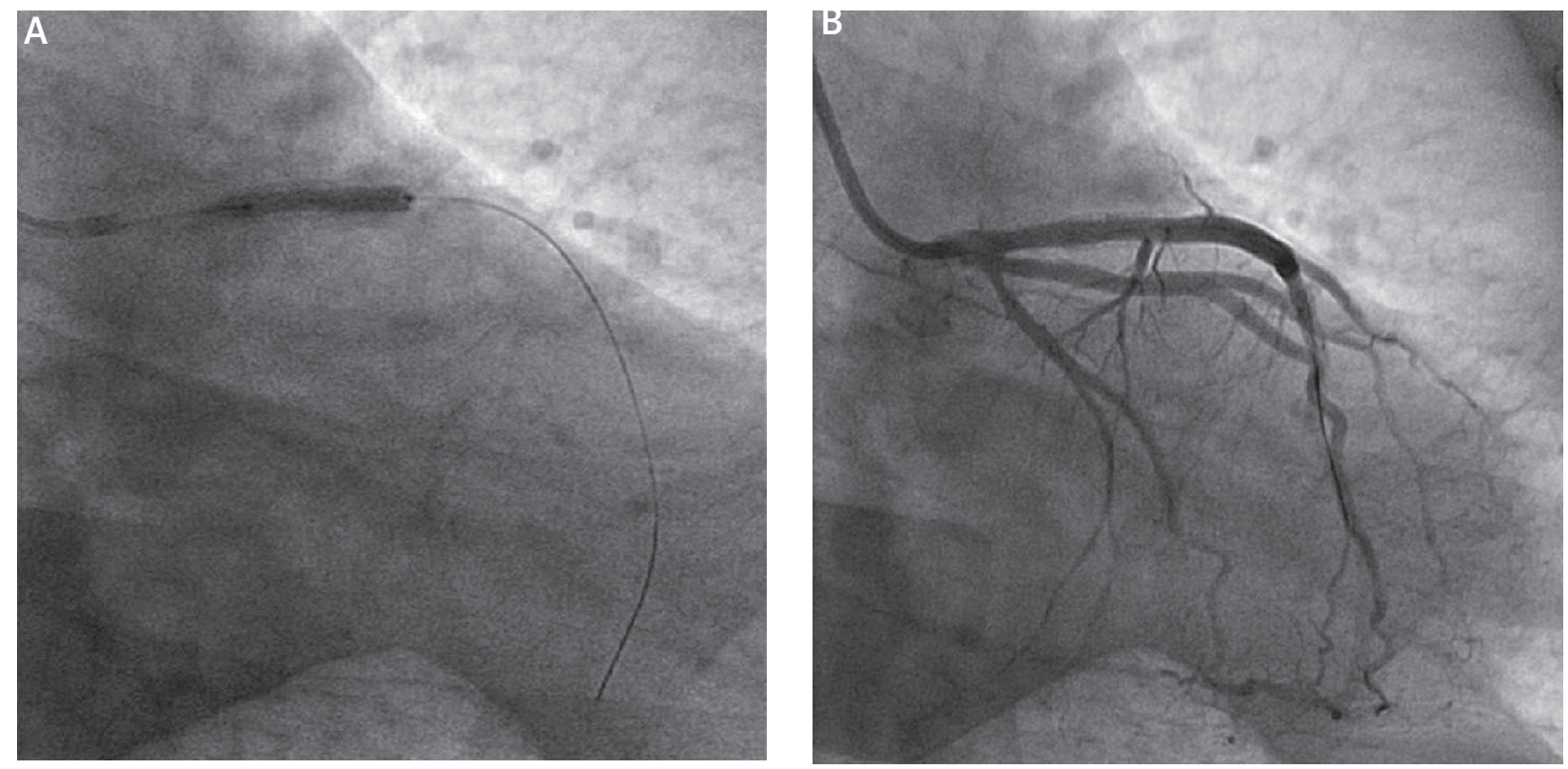

Figure 2. Cutting balloon dilatation (A) and resorption of in-stent plaque after pre-dilatation (B)
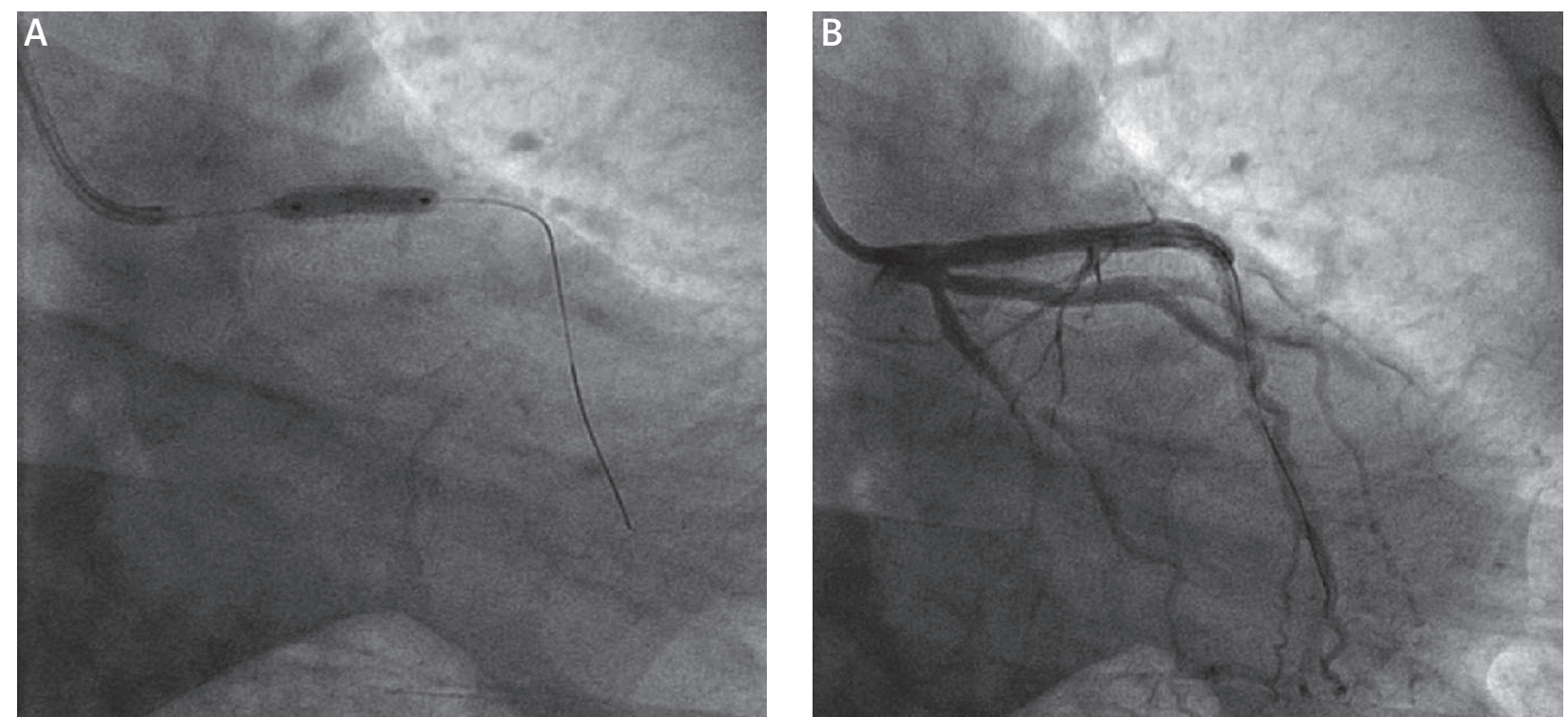

Figure 3. BVS deployment (A) and final image (B). Note that the final image of the proximal left anterior descending artery resembles the image taken after cutting balloon dilatation

balloon at the maximal proposed pressure (Figure 2; note dense and diffuse plaque burden resisting the predilatation), and a $3.5 \times 18$ BVS (Absorb, Abbot Vascular) was deployed, also with maximal proposed pressure. After post dilation of BVS with a non-compliant balloon, the procedure was terminated with a successful result (Figure 3).

\section{Discussion}

Bioresorbable vascular scaffolds have different mechanical properties as compared to metallic stents. Thus, the standard procedural technique to achieve appropriate deployment may differ [2]. Incomplete scaffold ap- position and uncovered struts are related to higher risk of scaffold thrombosis [3]. Therefore, quantitative angiography with optical coherence tomography (OCT) or intravascular ultrasounds (IVUS) is preferred in order to achieve optimal apposition [3, 4]. Usage of BVS in complex coronary lesions is still challenging because of the unique texture [5]. Utilisation of debulking techniques, including cutting balloon and directional atherectomy prior to BVS deployment, is also questionable. Herein, we show that prior cutting balloon dilatation is useful and may facilitate optimal BVS apposition. Lack of OCT or IVUS analysis is a major criticism of our proposal. Howev- 
er, the similarity of both images taken after cutting balloon dilatation and BVS deployment may serve as reliable supportive evidence for our statement. The significance of the routine use of a debulking technique prior to BVS deployment has no yet been reported. Further series and studies may clarify these statements more clearly.

\section{Conclusions}

Lesion preparation before BVS deployment is a crucial point. Use of a debulking technique including cutting balloon predilatation may facilitate optimal BVS apposition.

\section{Conflict of interest}

The authors declare no conflict of interest.

\section{References}

1. Oberhauser JP, Hossainy S, Rapoza RJ. Design principles and performance of bioresorbable polymeric vascular scaffolds. Eurolntervention 2009; 5 Suppl F: F15-22.

2. Gomez-Lara J, Diletti R, Brugaletta S, et al. Angiographic maximal luminal diameter and appropriate deployment of the everolimus-eluting bioresorbable vascular scaffold as assessed by optical coherence tomography: an ABSORB cohort B trial sub-study. Eurolntervention 2012; 8: 214-24.

3. Gomez-Lara J, Radu M, Brugaletta S, et al. Serial analysis of the malapposed and uncovered struts of the new generation of everolimus-eluting bioresorbable scaffold with optical coherence tomography. JACC Cardiovasc Interv 2011; 4: 992-1001.

4. Brugaletta S, Radu MD, Garcia-Garcia HM, et al. Circumferential evaluation of the neointima by optical coherence tomography after ABSORB bioresorbable vascular scaffold implantation: can the scaffold cap the plaque? Atherosclerosis 2012; 221: 106-12.

5. Meincke F, Kuck KH, Bergmann MW. Delivery of a bioresorbable vascular scaffold to complex lesions. Catheter Cardiovasc Interv 2014; 84: 774-8. 